\title{
Multiresolution Analysis of Tidal Debris Structures in Groups of Galaxies
}

\author{
D. N. Epitácio Pereira ${ }^{1}$, A. B. De Mello ${ }^{1}$, \\ and C. R. Rabaça ${ }^{2}$ \\ ${ }^{1}$ Departamento de Astronomia, Observatório Nacional, \\ R. Gal. José Cristino 77, 20921-400, Rio de Janeiro/RJ, Brazil \\ ${ }^{2}$ Observatório do Valongo, Universidade Federal do Rio de Janeiro, \\ Ladeira do Pedro Antônio 43, 20080-090 Rio de Janeiro/RJ, Brazil
}

\begin{abstract}
Galaxies in groups and clusters often experience strong tidal forces from its neighbors, expelling a significant amount of matter (stars and gas) to the surrounding environment, giving rise to 'tidal debris' features. We present the first experiments in a program of identification of substructures of extragalactic tidal debris elements in images from the HST archive, using wavelet-based multiresolution analysis. Tidal structures in two compact groups of galaxies, HCG 79 (Seyfert's Sextet) and HCG 92 (Stephan's Quintet) have been analyzed so far.
\end{abstract}

Keywords. techniques: image processing, galaxies: clusters: individual (HCG 79, HCG 92)

The multiresolution analysis approach consists in obtaining the representation of an image in multiscale space (with position and size as basis) and performing all relevant processing in this representation. We use a new multiresolution image processing system (Epitácio Pereira et al., in preparation) based on the à trous algorithm for the discrete wavelet transform (for details see, for example, Starck et al. (1998)). We perform multiscale reconstruction of the objects in the images, producing individual images for each of the main components of the tidal structures - both extended and point sources.

We selected the tidal debris structures of HCG 79 and HCG 92 as the first targets of our project. From the debris in HCG 79 we reconstructed a main diffuse component and several overlapping smaller sources (sub-objects). On the diffuse component, we found two 'bridges' that connect the debris to the neighboring galaxy HCG79b. Some of the sub-objects are located along these bridges. In the images of HCG 92 we studied the "new tail' (described by Sulentic et al. (2001)), and obtained individual images for the main sub-objects of the structure. Photometry and geometry of the detected components will be presented in a future paper, along with analyses of additional groups.

\section{Acknowledgements}

Some of the data used in this work were obtained from the Multimission Archive at the Space Telescope Science Institute (MAST). STScI is operated by the Association of Universities for Research in Astronomy, Inc., under NASA contract NAS5-26555. Support for MAST for non-HST data is provided by the NASA Office of Space Science via grant NAG5-7584 and by other grants and contracts.

\section{References}

Starck, J.-L., Murtagh, F. \& Bijaoui, A. 1998, Image processing and data analysis. The multiscale approach, Cambridge University Press.

Sulentic, J. W., Rosado, M., Dultzin-Hacyan, D., Verdes-Montenegro, L., Trinchieri, G., Xu, C. \& Pietsch, W. 2001, AJ, 122, 2993. 